\title{
Revisiting experimental methods for studies of acidity- dependent ocean sound absorption
}

\author{
Timothy F. Duda ${ }^{\text {a) }}$ \\ AOPE Department, MS 11, Woods Hole Oceanographic Institution, Woods Hole, Massachusetts 02543
}

(Received 28 October 2008; revised 5 February 2009; accepted 6 February 2009)

\begin{abstract}
The practical usefulness of long-range acoustic measurements of ocean acidity-linked sound absorption is analyzed. There are two applications: Determining spatially-averaged $p \mathrm{H}$ via absorption measurement and verifying absorption effects in an area of known $p \mathrm{H}$. The method is a differential-attenuation technique, with the difference taken across frequency. Measurement performance versus mean frequency and range is examined. It is found that frequencies below 500 $\mathrm{Hz}$ are optimal. These are lower than the frequency where the measurement would be most sensitive in the absence of noise and signal fluctuation (scintillation). However, attenuation serves to reduce signal-to-noise ratio with increasing distance and frequency, improving performance potential at lower frequencies. Use of low frequency allows longer paths to be used, with potentially better spatial averaging. Averaging intervals required for detection of fluctuations or trends with the required precision are computed. (C) 2009 Acoustical Society of America.
\end{abstract}

[DOI: 10.1121/1.3089591]

PACS number(s): 43.30.Pc, 43.30.Es, 43.30.Cq [RCG] Pages: 1971-1981

\section{INTRODUCTION}

Here, a multiple-frequency acoustic method to measure ocean $p \mathrm{H}$-dependent sound absorption is reinvestigated. The method was first investigated by Jin and Worcester (1989). Using knowledge of how $p \mathrm{H}$ and absorption are related (Mellen and Browning, 1977; Brewer et al., 1995), the method can be used either to determine spatially-averaged $p \mathrm{H}$ or to investigate details of absorption behavior if $p \mathrm{H}$ is known. Details regarding the first of these uses are the main focus of this paper, although the expressions can be modified for the reverse investigation. Applying the method as an acidity measure, ocean $p \mathrm{H}$ and temporal $p \mathrm{H}$ variations are estimated from the difference of sound attenuation at two (or more) frequencies. Being more specific, the technique would provide a nearly instantaneous remote measurement of sound absorption along an acoustic path, inferred mean $p \mathrm{H}$ along the path, and possibly a mean $p \mathrm{H}$ depth-profile in a slice filled by a set of acoustic paths oscillating vertically in the ocean acoustic waveguide. For studies of the temporal behavior of biogeochemical conditions over large physical scales, such an averaged $p \mathrm{H}$ measurement may (or may not) have sampling advantages over accumulated point measurements of $p \mathrm{H}$ in the situation of evolving ocean acidity having high-frequency or small-scale variability.

Global-mean ocean acidity must reflect future changes in atmospheric carbon dioxide concentration, which is rising, via flux of carbon dioxide into the ocean (see Doney et al., 2009a). An estimate of the average surface ocean water $p \mathrm{H}$ reduction from preindustrial times to 1996 is 0.1 unit (Brewer, 1997; Orr et al., 2005). This trend is predicted to continue, with further reduction by $0.3-0.5$ units possible in the next 100 years (Caldeira and Wickett, 2005; Orr et al.,

${ }^{a)}$ Electronic mail: tduda@whoi.edu
2005) with consequences to biogeochemical systems (see Sec. II). Because sound absorption is inversely related to $p \mathrm{H}$, it is possible that the ocean may become sonically noisier, all else constant (Hester et al., 2008). Measurements of global $p \mathrm{H}$ conditions are thus desirable. If trends are spatially broad and temporally smooth, infrequent and sparse localized $p \mathrm{H}$ measurements would adequately detect the conditions. If there are significant small-scale variations as suggested by Doney et al. (2009b), then more frequent and comprehensive measurements would be required to detect trends amidst the fluctuations.

Measurements of average large-scale $p \mathrm{H}$ conditions are typically made by accumulating point measurements along transects (Fig. 1) via programs such as the World Ocean Circulation Experiment (WOCE), the Joint Global Ocean Flux Study, and follow-up studies, or with time series (Fig. 2). More frequent or spatially-averaged measurements could be made with the acoustic method, if required. An acoustic measure of $p \mathrm{H}$ would have less accuracy and precision than individual point measurements but may provide equally valid mean estimates in the case of considerable variability of the individual measurements.

The acoustic measurement methodology for $p \mathrm{H}$-dependent absorption has been established by prior research (Jin and Worcester, 1989; Brewer et al., 1995). However, thorough analysis and implementation have lagged theoretical and technical readiness. Recent advances in ocean acoustic systems and in observations of long-range signal fluctuations (which add uncertainty to the measurement) make this a good time to revisit this technology. Inexpensive and reliable sound projectors are available at frequencies of 200-600 Hz. These are suitable for long-term use at ocean observatories that are coming online, along with highly capable receiver systems assembled from off-the-shelf components. In light of this new technology and new interest in possible trends in ocean acidity, this paper builds on the 

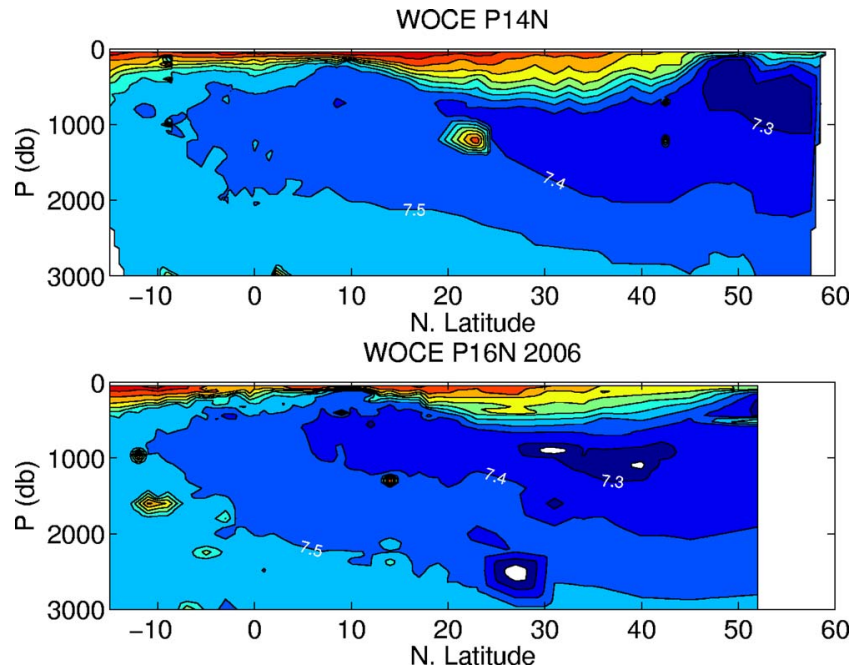

FIG. 1. (Color online) Full sections of $p \mathrm{H}$ in the tropical and North Pacific are shown. Data are from two WOCE transect cruises: P14 at longitude 180 in 1993 and P16 at longitude $150 \mathrm{~W}$ (approximately) in 2006. The contour interval is 0.1 . The 7.3, 7.4, and 7.5 contours are labeled. Shading is similar to Fig. 2. Data are from the Carbon Dioxide Information Analysis Center (CDIAC, 2009a).

analysis of Jin and Worcester (1989) by investigating the measurement capability at many frequencies, bandwidths, and propagation distances. The mean acoustic energy differ-
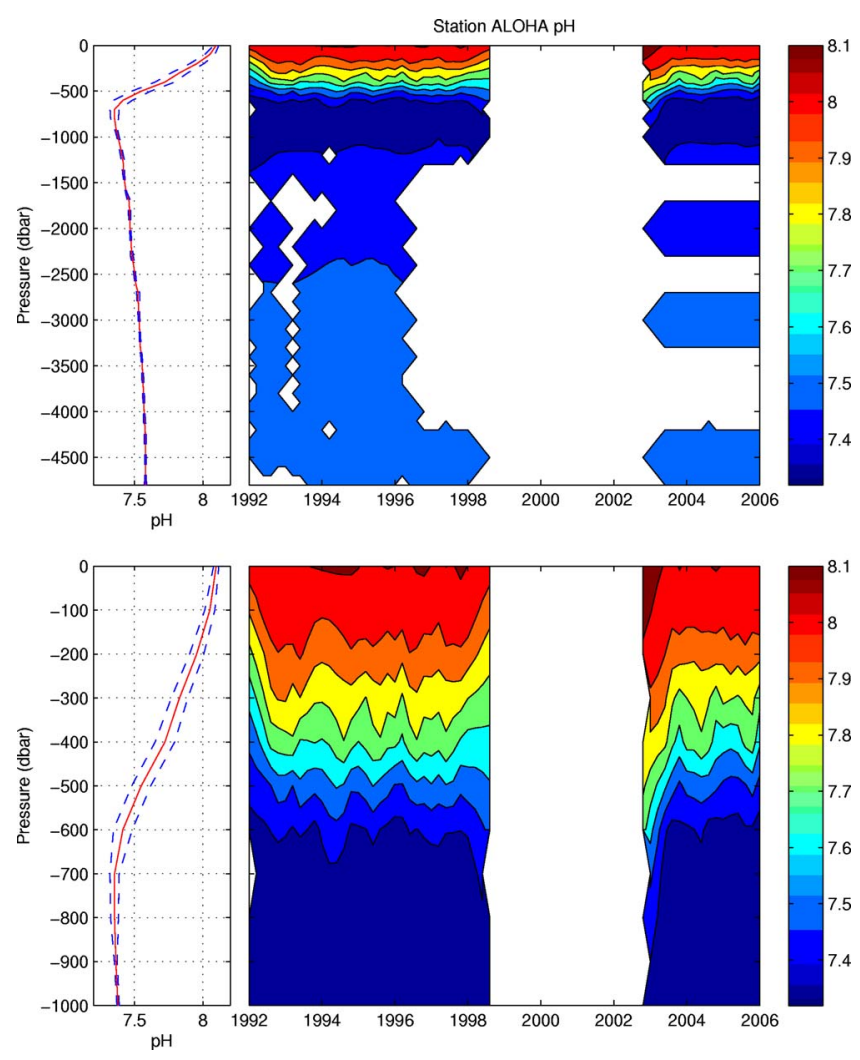

FIG. 2. (Color online) HOT Station ALOHA (2245' N, $158 \mathrm{~W}) p \mathrm{H}$ data collected over a 14 year period. Data are from University of Hawaii (2009), tabulated and plotted by Bruce Howe. The data are collected several times per year. At the left are the time-mean profiles plotted as solid lines, with plus/minus standard deviation shown with dotted lines. To the right the time series are contoured, contour interval 0.1 , with year indicated at bottom. The lower panels show an expanded view of the near-surface data, and the upper panels show all depths. ence of two (or more) frequencies must be determined after propagation over fairly long distances of a few hundred kilometers, but such propagation degrades signal-to-noise ratio and introduces variability via scattering from mesoscale, internal wave, and surface wave phenomena. In this paper, signal averaging requirements necessary to achieve useful results are analyzed.

The methodology can be described very simply. Research has established the relationship between aqueous boric acid-borate equilibrium and energy absorption of sound below $1 \mathrm{kHz}$ (Fisher and Simmons, 1977), although some anomalous behavior has been identified which suggests some interaction between borate and other ions occurs in the ocean (Mellen et al., 1981, 1983). Pressure-sensitive reactions of the relevant ions modify the phase lag between pressure and velocity in sound waves, leading to energy loss. This is linked to $p \mathrm{H}$ because the concentration of hydroxide $\mathrm{OH}^{-}$ (Mellen and Browning, 1977) and/or carbonate $\mathrm{CO}_{3}{ }^{2-}$ (Mellen et al., 1983) plays a role. The kinetic behavior of the slowest reaction introduces a time scale of about a $100 \mathrm{~ms}$, giving the $1 \mathrm{kHz}$ transition frequency (Zeebe et al., 2001). At high frequencies sound absorption is dominated by other processes and is not strongly dependent on $p \mathrm{H}$, whereas below $10 \mathrm{kHz}$ it is strongly $p \mathrm{H}$ dependent. This makes the shape of the absorption curve in the transition region, centered at about $600 \mathrm{~Hz}, p \mathrm{H}$-dependent in a manner that allows $p \mathrm{H}$ to be estimated from the shape of the curve. The absolute absorption at low frequency is also $p \mathrm{H}$ dependent, but can be difficult to measure because of uncertain net scattering loss. The shape of the absorption curve, determined by comparing signal level at two or more frequencies, is more sensible to study experimentally than absolute absorption because many propagation and scattering processes also affecting signal level are weak functions of frequency. That is, differencing over frequency minimizes detrimental scattering effects if they are weak functions of frequency (Jin and Worcester, 1989).

The differential technique can reduce or eliminate interference from mesoscale and weak internal-wave scattering, as long as such scattering does not cause wavefronts to break up into discernable micro-multi-path arrivals. However, some internal-wave induced fluctuations will exist, caused by interfering micro-fronts, and must be averaged over. Jin and Worcester (1989) recognized this as a major concern. They estimated the number of independent samples needed to provide useful measurements of $p \mathrm{H}$ (better than $0.05 \mathrm{ac}-$ curacy) to lie in the range of 44-100 for the case of 450-650 $\mathrm{Hz}$ signals propagated $750 \mathrm{~km}$, with sample independence attained when sample spacing exceeds the acoustic fluctuation time scale of tens of minutes. Results from internalwave induced fluctuation research over the past two decades are incorporated here into a new analysis to re-determine the best frequencies to use and the number of samples needed. It is found that slightly lower frequencies are better, and that many more than the previous estimate of 100 samples may be needed.

The methodology is reviewed here from beginning to end, and the sampling requirements re-examined. First, motivation for ocean acidity and sound absorption studies is 
discussed (Sec. II). Next, the physical chemistry of the problem is reviewed (Sec. III). In Sec. IV, the sensing method using long-range propagation is outlined [Jin and Worcester (1989) redux]. Section V examines the effectiveness of various frequency bands and path lengths in an idealized situation with steady acoustic propagation conditions and additive noise. Section VI shows how the probable presence of signal fluctuations dramatically increases the number of required samples that would need to be averaged together to obtain good results. Section VII summarizes the work.

\section{MOTIVATION}

Global ocean $p \mathrm{H}$ is likely to decrease because of ocean uptake of carbon dioxide deposited by society into the atmosphere. It is accepted that global surface $p \mathrm{H}$ has been reduced from 8.2 to 8.1 in the past 200 years (Brewer, 1997). Models predict that the surface $p \mathrm{H}$ will continue to drop as the atmospheric carbon dioxide concentration increases (Caldeira and Wickett, 2005). Local conditions such as vertical $p \mathrm{H}$ profiles throughout the world may be less amenable to prediction. A notable implication is that probable future inorganic carbon uptake by the ocean and oceanic $p \mathrm{H}$ reductions have the potential to profoundly affect calcium carbonate shelled or skeleton-producing animals, including (but not limited to) small pteropods, coral, clams, and lobsters (e.g., Feely et al., 2004; Orr et al., 2005, and references cited therein).

Changes in ocean $p \mathrm{H}$ whether from anthropogenic $\mathrm{CO}_{2}$ or from other causes are equally important acoustically. The acid level responds to other factors besides uptake of carbon dioxide, such as decreased ventilation from changes in physical climate, many of which are also likely to reduce $p \mathrm{H}$ (Hester et al., 2008). A simplified view of the carbon uptake effect is given: Aqueous carbon dioxide combines with water to form carbonic acid, which can dissociate into bicarbonate and hydrogen ions, or carbonate and two hydrogen ions. Because bicarbonate is very soluble, hydrogen ions and available carbonate ions will tend to form bicarbonate, which buffers the system against $p \mathrm{H}$ drop. However, this reduces carbonate concentration, which has the detrimental effect on calcium carbonate mineral structures. Even in water remaining supersaturated with respect to calcium carbonate, decreased calcification rates are expected (Orr et al., 2005).

Such $p \mathrm{H}$ and carbonate changes would affect localized ecosystems at coastal margins (Feely et al., 2008; Wootton et al., 2008) as well as pelagic ecosystems and their fisheries such as tuna. Coastal ecosystem alteration would have significant natural and economic impact, and may indeed be closely tied to what is happening offshore. Therefore, both coastal and pelagic situations are of interest. The deep-sea ocean volume in some sense sets the boundary condition for regional acidity, and may serve as a reservoir or be a driver of change. Orr et al. (2005) and Feely et al. (2004) showed that subpolar regions are likely to be the first to reach undersaturation of calcium carbonate minerals calcite and aragonite, with probable pronounced biological impacts in those important areas.
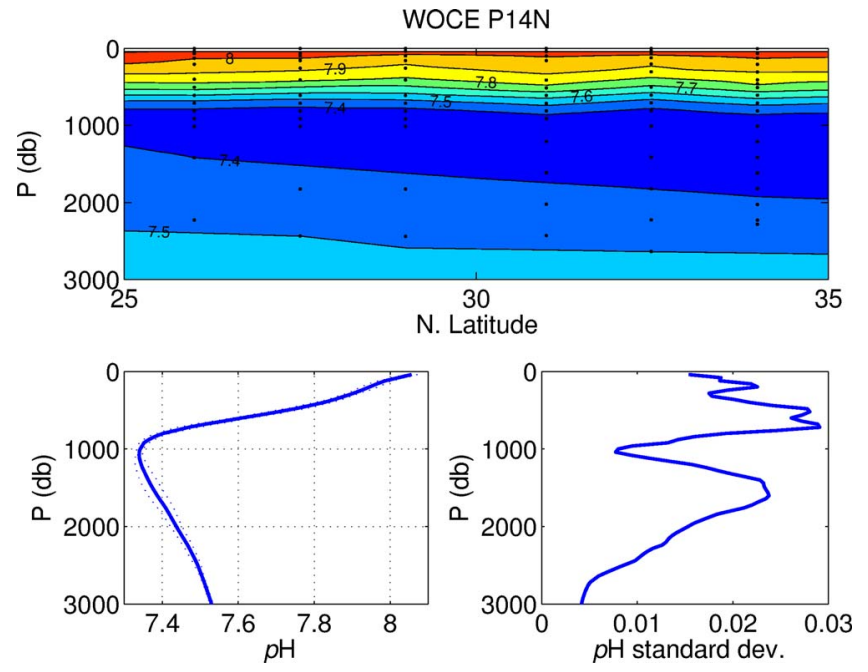

FIG. 3. (Color online) A portion of WOCE transect P14N from 1993 (Fig. 1 ) is shown in the top panel. The longitude is approximately $180^{\circ}$. The mean profile is shown at lower left, and the standard deviation profile at lower right. The dots in the upper panel show the locations of the data points. Data are from the Carbon Dioxide Information Analysis Center (CDIAC, 2009a).

Tracking $p \mathrm{H}$ in certain areas over time with effective averaging of short-term and small-scale variability may be desirable to assess the progress of these changes. However, representative measures of average deep-sea $p \mathrm{H}$ using ships are expensive. Sections of oceanic $p \mathrm{H}$ show mesoscale spatial and temporal variations (Fig. 1), which must be averaged over time and space in order to determine mean values and trends. At least two papers show scatter plots of oceanic $p \mathrm{H}$ from hydrographic cruises with fluctuations of \pm 0.05 about a mean profile (Brewer et al., 1995; Ternon et al., 2001) illustrating the natural variability and possible sampling problem. Hawaii Ocean Timeseries (HOT) measurements show similar variability (Fig. 2), as do transects of $p \mathrm{H}$ (Fig. 3).

There are many features of the global carbonate system that may or not be stable. An example is the subsurface tongue of low $p \mathrm{H}$ seen in Figs. 1 and 3. This is associated with low surface $p \mathrm{H}$ in the subpolar region associated with carbon dioxide flux into the ocean at those higher latitudes due in part to thermodynamic effects (Takahashi et al., 2006). The feature is consistent with low alkalinity tongues descending from the subpolar North Pacific in hydrography sections (CDIAC, 2009b). Similar features occur in the South Atlantic and South Pacific but not in the North Atlantic.

A full examination of $p \mathrm{H}$ variability in the Atlantic Ocean is available (Ternon et al., 2001). The work shows that $p \mathrm{H}$ can be predicted from various local parameters (temperature, salinity, oxygen concentration, nitrate concentration, etc., not including carbon dioxide and carbonate) but only to within roughly 0.02 .

Note that reduced ocean $p \mathrm{H}$ would mean reduced absorption of sound below a few kilohertz, possibly causing the ocean to become noisier at those frequencies, all else equal (Hester et al., 2008).

One approach toward quantifying trends in oceanic $p \mathrm{H}$, and associated sound absorption, and testing our ability to predict them would be implementation of a frequency- 
dependent acoustic absorption system in the deep sea [Jin and Worcester (1989) type]. An acoustic system could automatically and make repeated instantaneous spatiallyaveraged measurements and may be able to make the absorption and $p \mathrm{H}$ measurements with useful precision and accuracy. This technique could prove cost effective with respect to ship-survey $p \mathrm{H}$ methods, particularly if implemented south of $40^{\circ} \mathrm{S}$, one of the subpolar areas at risk of profound carbonate biogeochemistry change. Other reasons for measuring the $p \mathrm{H}$ of the pelagic ocean are that pelagic $p \mathrm{H}$ modeling may be more robust than in coastal regions and may be at the point of testability, and that spatial averaging over the known scales of natural variability with long-range acoustics is straightforward. The acoustic measurement resolution limit for $p \mathrm{H}$ will be shown to be about 0.05 , larger than the variations shown in Figs. 2 and 3 for the central North Pacific, and thus would be unable to improve upon transect-mean estimates from areas having comparable variability. The acoustic technique would be most useful in areas with higher spatial variability or strong temporal variability.

\section{III. $p H$-DEPENDENT SOUND ABSORPTION FROM BORATE RELAXATION}

A solution containing the borate ion $\left(\mathrm{B}(\mathrm{OH})_{4}^{-}\right)$and boric acid $\left(\mathrm{B}(\mathrm{OH})_{3}\right)$, present in small quantities in ocean water, changes volume when the dissolved boric acid/borate changes between three states, the end members being boric acid and borate. The volume change causes absorption as described below. Many coupled reactions may occur in concert with the borate reactions. A concise recent summary of uncertainties regarding the chemistry appears in Hester et al., 2008. The simplest candidate reaction that may result in sound absorption is

$$
\mathrm{B}(\mathrm{OH})_{3}+\mathrm{OH}^{-} \leftrightarrow \mathrm{B}(\mathrm{OH})_{3} \cdot \mathrm{OH}^{-} \leftrightarrow \mathrm{B}(\mathrm{OH})_{4}^{-}
$$

(Mellen and Browning, 1977; Mellen et al., 1980), which does not involve other coupled reactions. This describes absorption data qualitatively but not quantitatively. Boric acid concentration exceeds borate at oceanic $p \mathrm{H}$. This process roughly explains the frequency dependence of absorption because the right-hand step has the slower time constant than the other (somewhere around a millisecond) and yields a reduction in volume. The equilibrium state of Eq. (1) is pressure-dependent, explaining why acoustic waves trigger the transient volume changes. The system thus has what might be loosely described as a dynamic compressibility, which gives sound-speed an imaginary component (Liebermann, 1949). The borate acoustic absorption with relaxation frequency in the $1 \mathrm{kHz}$ range was identified in the laboratory (Yeager et al., 1973). That work followed earlier work involving salts with higher relaxation frequencies, primarily magnesium sulfate. Another system of reactions with similar absorption behavior is

$$
\begin{gathered}
\mathrm{H}_{2} \mathrm{O}+\mathrm{B}(\mathrm{OH})_{3}+\mathrm{OH}^{-}+\mathrm{CO}_{3}{ }^{2-} \leftrightarrow \mathrm{B}(\mathrm{OH})_{4}{ }^{-}+\mathrm{HCO}_{3}{ }^{-}+\mathrm{OH}^{-} \\
\uparrow \\
\mathrm{H}_{2} \mathrm{O}+\mathrm{B}(\mathrm{OH})_{3} \cdot \mathrm{OH}^{-}+\mathrm{CO}_{3}{ }^{2-} \leftrightarrow \mathrm{B}(\mathrm{OH})_{4}{ }^{-}+\mathrm{H}_{2} \mathrm{O}+\mathrm{CO}_{3}{ }^{2-}
\end{gathered}
$$

(Mellen et al., 1983). This is a reduced notation for a few possible systems that include pairing of some of the ions with metallic ions. The horizontal steps are the slow ones associated with low-frequency absorption. This system was proposed to account for absorption differences between solutions of borate alone and artificial seawater solutions containing dissolved inorganic carbon. Both Eqs. (1) and (2) involve $p \mathrm{H}$ via the role of the $\mathrm{OH}^{-}$concentration.

The dissolution of $\mathrm{CO}_{2}$ in the ocean and the subsequent formation of carbonic acid $\left(\mathrm{H}_{2} \mathrm{CO}_{3}\right)$ change the concentration of hydroxide $\left(\mathrm{OH}^{-}\right)$by releasing protons and forming three ions: bicarbonate $\left(\mathrm{HCO}_{3}{ }^{-}\right)$, carbonate $\left(\mathrm{CO}_{3}{ }^{2-}\right)$, and hydronium $\left(\mathrm{H}_{3} \mathrm{O}^{+}\right.$, equivalent for our purposes to protons, $\left.\mathrm{H}^{+}\right)$. (This changes the $p \mathrm{H}$, the negative of the base-10 logarithm of hydronium concentration.) The hydronium ions react with the $\mathrm{OH}^{-}$ions in the alkaline ocean to form water, changing the $\mathrm{OH}^{-}$concentration, and thus also changing the relative concentrations of boric acid and borate, which alters the absorption because the transient reactions of Eq. (1) and/or Eq. (2) proceed at levels determined by the concentrations. In the weakly alkaline ocean, the reagent in Eq. (1) or Eq. (2) with least concentration is the hydroxide ion, so the reaction is closely coupled to its concentration. As a result, absorption is an increasing function of $p \mathrm{H}$ (decreasing function of acidity). This $p \mathrm{H}$ dependency is independent of the sum concentration of borate and boric acid, which is essentially proportional to salinity.

Two additional chemical species cause absorption, magnesium sulfate and magnesium carbonate, but they have higher relaxation frequencies. Their absorptions are weak with respect to that of borate and are essentially constant with respect to frequency and $p \mathrm{H}$ at the low frequencies of interest here.

\section{DIFFERENTIAL-ATTENUATION MEASUREMENTS OF ABSORPTION}

Using notation in Brewer et al. (1995), the coefficient of sound absorption (units $\mathrm{dB} / \mathrm{km}$ ) can be written as

$$
\alpha(f)=\alpha_{b}(f)+\alpha_{m}(f)+\alpha_{c}(f) .
$$

Below $1 \mathrm{kHz}$ the $p \mathrm{H}$-sensitive borate term $\alpha_{b}$ dominates. Below $700 \mathrm{~Hz}$ the other terms (magnesium sulfate $\alpha_{m}$ and magnesium carbonate $\alpha_{c}$ ) can be neglected (Fisher and Simmons, 1977). Signal power in decibels is defined as a ratio $P=10 \log _{10}\left(I / I_{0}\right)$, where $I$ is intensity (peak amplitude squared for a sine wave) and $I_{0}$ is a reference value. The borate term can be written as

$$
\alpha_{b}=(S / 35) A_{b}\left(f^{2} f_{b} /\left(f^{2}+f_{b}^{2}\right)\right),
$$

where $A_{b}=0.1 \mathrm{~K}, f_{b}=0.9 \times 10^{T / 70} \mathrm{kHz}, S$ is salinity (psu), and $T$ is temperature $\left({ }^{\circ} \mathrm{C}\right)$. The $p \mathrm{H}$-dependence (NBS $p \mathrm{H}$ scale) is contained in $K=10^{(p \mathrm{H}-8)}$. Figure 4 shows borate absorption versus frequency for various $p \mathrm{H}$ values at fixed salinity and temperature. The measurement of absorption (and thus of $p \mathrm{H}$ if the other parameters are sufficiently well known) cannot be reliably made by measuring signal strength at one frequency in the ocean because acoustic forward scattering processes from mesoscale eddies, surface en- 


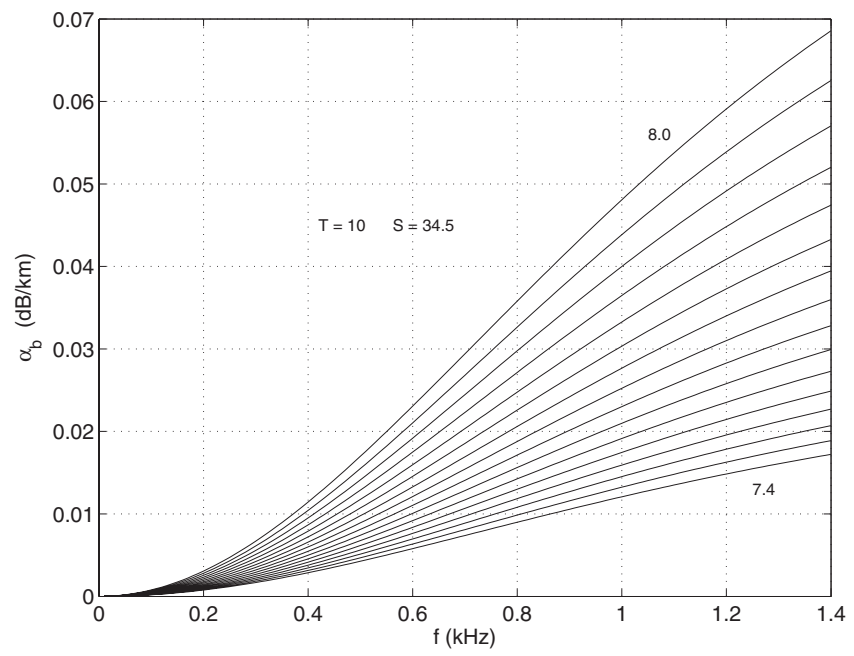

FIG. 4. Borate absorption $\alpha_{b}$ as a function of frequency is plotted for temperature $10^{\circ}$ and salinity 34.5 for $p \mathrm{H}$ from 7.4 to 8.0 by 0.04 increments. Total absorption values at $1 \mathrm{kHz}$ are $18 \%$ higher $(p \mathrm{H} 8.1)$ to $65 \%$ higher $(p \mathrm{H}$ 7.4) than the borate absorption.

counter, and seabottom encounter cause large fluctuations in energy. However, $p \mathrm{H}$-dependent sound absorption due to borate is a function of frequency, so that the fingerprint of this process can be obtained using energy measurement at two or more frequencies, enabling recovery of $p \mathrm{H}$. This assumes that scattering loss differences between the frequencies are small. For the frequencies investigated here, this is most questionable for surface reflecting rays in high sea states (Erbe and Farmer, 2000; Brekhovskikh and Lysanov, 1982). Such rays may be the only type available for long-range paths in polar regions; in other areas fully refracted rays should be available.

Loosely following Jin and Worcester (1989) but altering the equations to include updated information in Brewer et al. (1995), the differential attenuation along a single propagation path $\Gamma$ with arc increment $d s$ is given by

$$
\begin{aligned}
D_{A}\left(f_{1}, f_{2}\right) & =\int_{\Gamma} \alpha\left(f_{2}\right) d s-\int_{\Gamma} \alpha\left(f_{1}\right) d s \\
& =\int_{\Gamma} \int(\partial \alpha(s) / \partial f) d f d s,
\end{aligned}
$$

where $f$ is frequency in kilohertz. The paths are the same, so after retaining only the dominant term $\alpha_{b}$,

$$
D_{A}=\int_{\Gamma}(S(s) / 35) A_{b}(s) f_{b}(s)\left(\frac{f_{2}^{2}}{f_{2}^{2}+f_{b}^{2}(s)}-\frac{f_{1}^{2}}{f_{1}^{2}+f_{b}^{2}(s)}\right) d s .
$$

The position-dependent terms (those dependent on $s$ ) are functions of either $T$ or $p \mathrm{H}$. If $p \mathrm{H}$ is known then measurement of $D_{A}$ would test the veracity of the expressions for $K$, $A_{b}$, and $f_{b}$. Alternatively, comprehensive inversion of a group of ray-integrals (6) using methods in Munk et al., 1995 would provide $S, K$ (thus $p \mathrm{H}$ ), and $f_{b}$ (thus $T$ ) as functions of position in a source-receiver vertical slice. However, acoustic data alone would probably be insufficient for a complete two-dimensional inversion. A reasonable approach more
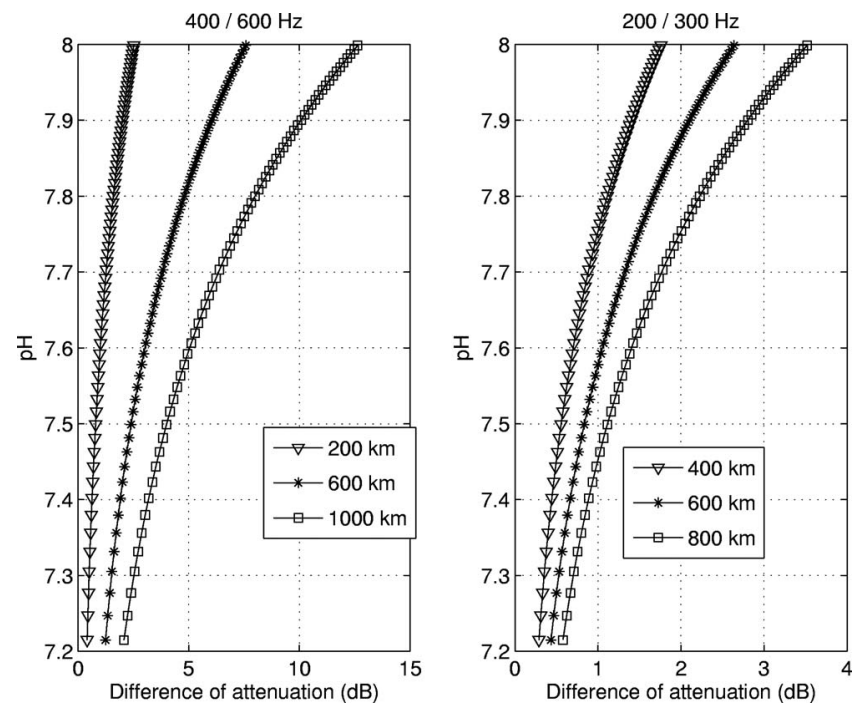

FIG. 5. $D_{A}$ versus $p \mathrm{H}$ computed using expression (10) is plotted for two different frequency pairs and a few propagation distances.

likely to yield reliable inversion would be to divide the integrals into a few parts each corresponding to depth increments, such as 0-100 m, 100-200 m, etc., and to incorporate auxiliary information to refine the solution (such as limiting $p \mathrm{H}$ fluctuations to the upper kilometer, for instance). Temperature structure (affecting $f_{b}$ ) is sufficiently well-known to allow inversion for $p \mathrm{H}$. A test-case published by Jin and Worcester (1989) derived a solution for a path that could be approximated with a constant $T$ equal to $4^{\circ}$. This is the temperature near the sound channel axis at lower latitude sites (such as HOT, Fig. 2). A constant low temperature assumption is also suitable for polar regions. At this temperature, the frequency $f_{b}=1 \mathrm{kHz}$ (note that $f_{b}$ increases to $2 \mathrm{kHz}$ at $24^{\circ}$ ), and integration gives the approximate solution

$$
D_{A}=A_{b} L C
$$

for $S$ equal to 35 , where $L$ is source-receiver distance in kilometers, and where

$$
C=\frac{f_{2}^{2}}{f_{2}^{2}+1}-\frac{f_{1}^{2}}{f_{1}^{2}+1} .
$$

From this, using the definition $A_{b}=0.0237 \times 10^{(0.83 p \mathrm{H}-6)}$, they obtain

$$
p \mathrm{H}=0.83^{-1}\left[6+\log _{10}\left(\frac{D_{A}}{0.0237 L C}\right)\right] .
$$

Using our definition of $A_{b}$ and letting $S$ vary gives the slightly different expression

$$
p \mathrm{H}=8+\log _{10}\left(\frac{350 D_{A}}{S L C}\right) .
$$

Figure 5 shows the differential absorption that must be measured at various frequencies and ranges to determine $p \mathrm{H}$ using this constant temperature approximation [i.e., using Eq. (10)]. Salinity of $35 \mathrm{psu}$ is assumed. The measurement sensitivity is given by $d(p H) / d D_{A}=\left(\ln (10) D_{A}\right)^{-1}=0.4343 / D_{A}$. From Fig. 5, at $600 \mathrm{~km}$ range, bandwidth of $200 \mathrm{~Hz}(400$, $600 \mathrm{~Hz}$ ), a $\Delta p H$ of 0.05 at $p \mathrm{H} 7.6$ yields $\Delta D_{A}$ of $0.58 \mathrm{~dB}$. 
Source and receiver depth placement can contribute to how Eq. (6) is utilized to investigate differential attenuation and/or $p \mathrm{H}$. Use of near-axial acoustic rays to determine $p \mathrm{H}$, which travel through water where $p \mathrm{H}$ and $T$ are relatively constant, as opposed to vertically cycling rays which integrate over vertically varying $T, p \mathrm{H}$, and thus $\alpha_{b}$, is the scenario leading to Eqs. (7) and (10), but those paths undergo much scattering and may have amplitude fluctuations that make them unusable (Duda et al., 1992; Colosi et al., 1994) (effects of scattering are discussed in Sec. VI). Polar regions also have near-constant temperature (a few ${ }^{\circ} \mathrm{C}$ variations), although the rays cycle vertically and reflect from the surface, which may add frequency-dependent surface loss. A full vertically-dependent inverse determination would be straightforward to implement and would show a slightly different relationship between distance, $D_{A}$, and mean $p \mathrm{H}$ than Eq. (10), but Eq. (10) is used as a guideline for measurement sensitivity in Sec. V, as done by Jin and Worcester (1989). Note that receivers at multiple depths in the oceanic sound channel on a single mooring would receive eigenray signals that may be spaced such that their internal-wave fluctuation effects are independent, adding reliability to the measurement. Section VI addresses how independent measurements must be used to build statistical reliability of the method.

In a full inversion, a depth profile of $p \mathrm{H}$ is a likely result. This procedure is analogous to temperature inversion, and would require differential attenuation to be measured along more than one acoustic eigenray. Simplification (i.e., depth function parametrization) may be helpful if the approximate depth profile of $p \mathrm{H}$ is known and has its shape constrained by known processes, or if the depth profile of $\mathrm{rms} p \mathrm{H}$ fluctuations is known (i.e., fluctuations limited to the near-surface region).

The crucial quantity $\alpha_{b}$ is $T$ - and $S$-dependent at fixed $p \mathrm{H}$, as shown. In situations where this constant $T$ approximation is usable, neglect of expected natural $T$ and $S$ variations along the acoustic path would lead to errors of not more than $4 \%$ in forward modeling of $D_{A}$. This would give errors smaller than 0.03 in $p \mathrm{H}$ computation from observed $D_{A}$.

\section{MEASUREMENT EFFECTIVENESS VERSUS FREQUENCY AND RANGE}

To satisfy the goal of making meaningful continuous spatially-averaged measurements, long measurement ranges are desired to average over mesoscale features. The primary acoustic data, $D_{A}$, grow with range and become more easily measurable. Thus, there are two reasons why long propagation paths are desirable. This would imply that low frequencies are necessary because of the prototypical frequency dependence of absorption, which is empirically in the Pacific, for example (disregarding details appearing in Sec. IV) (Munk et al., 1995)

$$
\alpha(f)=0.79 A \frac{f^{2}}{0.8^{2}+f^{2}}+\frac{36 f^{2}}{5000+f^{2}} \quad \mathrm{~dB} / \mathrm{km},
$$

where $f$ again has units of kilohertz and the geographically variable quantity $A$ is 0.055 for the North Pacific. On the other hand, the measurement sensitivity to frequency,

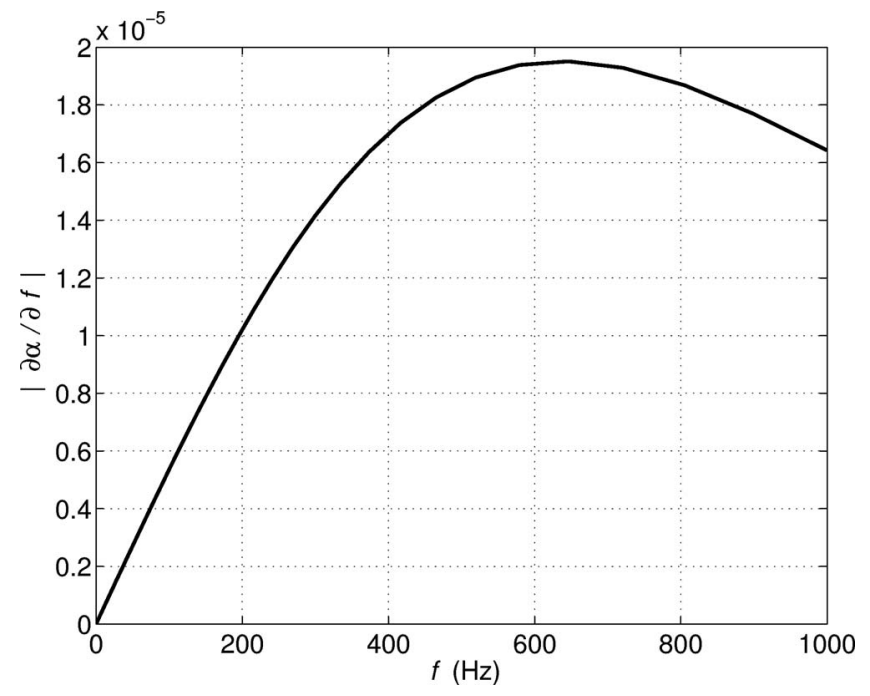

FIG. 6. The derivative of the absorption curve is shown.

$|\partial \alpha / \partial f|$, related to $D_{A}$, has a peak near $0.6 \mathrm{kHz}$ (Fig. 6), apparently diminishing the effectiveness of lower frequencies, as reasoned by Jin and Worcester (1989). This is shown here to not be the entire story, with a balance occurring between low differential attenuation at low frequency and short range, and low signal strength at high frequency and long range.

\section{A. Signal method}

The system design assumed in this performance analysis section is one capable of making acoustic measurements of discrete eigenray arrivals (wavefront segments, equivalently) that are common to two (or more) frequency bands. This can involve looking at the band-edges of a wideband signal (as with Jin and Worcester, 1989), which would have lower signal to noise than the band center, or it can involve looking at two independent bands from either one source or two sources. The amplitude difference is taken to obtain $D_{A}$. In addition to occupying two or more bands, the signals in each band must have enough bandwidth to provide good coherent processing gain via pulse-compression techniques (Munk et al., 1995). This is required to improve signal-to-noise ratio and to identify acoustic ray geometry if full inversion of Eq. (6) is desired. Signal strength measurements (i.e., attenuation) would be made using peak amplitudes of pulsecompression output for isolated identified eigenrays. Alternatively, spectral level of the pulse-compression output for the isolated arrivals could be used, which would correspond to a base-band version of the received signal spectrum with significant signal power gain, with gain given by the ratio of the total signal duration divided by the inverse signal bandwidth (or code digit length). Ideally, signals in each band would be transmitted at the same time from very closely spaced sources. The best situation would be to use one source with a very broad bandwidth, more than $300 \mathrm{~Hz}$. 


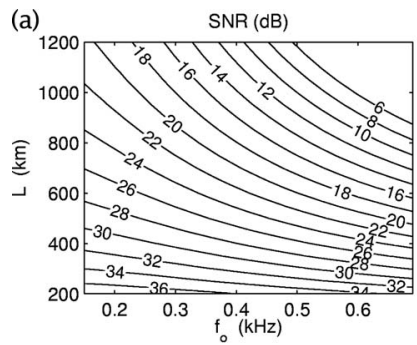

(b)

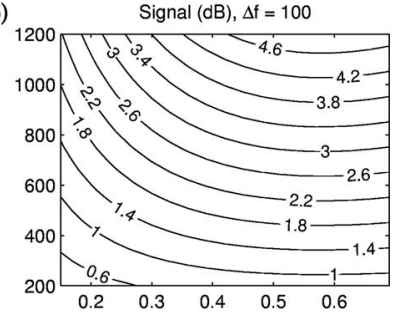

(c)

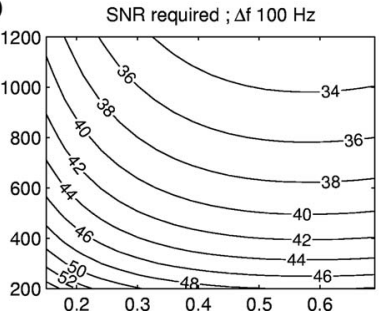

(d)

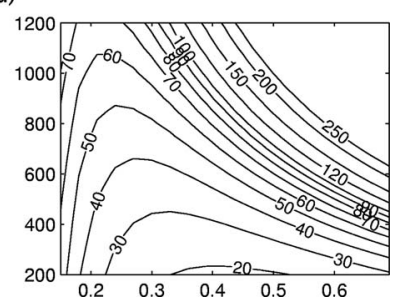

FIG. 7. Four plots display contours of four different quantities versus center frequency and path length $L$ of a dual-frequency differential-attenuation system. (a) SNR from formula (12) is shown. $\mathrm{PG}=24 \mathrm{~dB}, \mathrm{SL}=185 \mathrm{~dB}$. (b) Signal level $D_{A}$ for $100 \mathrm{~Hz}$ frequency difference, $p \mathrm{H}=7.8$ is shown. (c) SNRR to achieve the $D_{A}$-scaled $\varepsilon$ requirement [from (b)] for detection of a $p \mathrm{H}$ difference of 0.05 . This is computed from Eq. (13) after determining the $\varepsilon$ requirements from the $D_{A}$ curves in (b). (d) The number of samples needed to achieve the required $\varepsilon$ (or SNRR) computed from information in panels (a) and (c) using Eq. (14b).

\section{B. Dependency of signal-to-noise ratio}

To determine the best frequency (without building an expression for optimum frequency at this time), begin with the sonar equation

$$
\operatorname{SNR}(f, L)=\mathrm{SL}-\mathrm{TLa}(f, L)-\mathrm{TLg}(L)-(\mathrm{NL}(f)-\mathrm{PG}) .
$$

The terms are signal-to-noise ratio (SNR), source level (SL), absorption transmission loss (TLa), geometrical spreading transmission loss (TLg), noise level (NL), and processing gain (PG) [PG is substituted for the conventional array gain (AG)]. Here, set source level $=185 \mathrm{~dB}$, noise level $=65 \mathrm{~dB}$ (moderate sea state), and $\mathrm{PG}=24 \mathrm{~dB}$ for a coded pulse. TLg is conservatively expressed as spherical spreading, which approximately accounts for the division of energy into multiple arrivals by having excess loss beyond that of cylindrical spreading that might better describe pure tone transmission. Figure 7(a) shows SNR for these parameters as a function of frequency and range. SNR decreases with frequency and range.

\section{Dependency of differential attenuation}

Figure 7(b) shows $D_{A}(f, L)$ from Eq. (7) modeled for $100 \mathrm{~Hz}$ separation. The signal $D_{A}$ increases with range and center frequency, except above $600 \mathrm{~Hz}$. Note the SNR and the signal $\left(D_{A}\right)$ have opposed trends in terms of suitability with respect to frequency and range.

An important aspect of the inversion problem is the accuracy of $D_{A}$ measurement. For oceanic transmission measurements, there are two effects which cause difficulty in measuring this difference of two mean signal levels. One is the fact that the measurement is composed of signal plus

noise, not just signal. The other is the frequency-dependence of natural signal fluctuations caused by interfering microfronts.

In the presence of Gaussian noise, Jin and Worcester (1989) showed that upper-bound of measurement error for signal strength, in decibels, is given by

$$
\varepsilon=20 \log _{10}\left(1+\sqrt{2} \times 10^{-\mathrm{SNRR} / 20}\right),
$$

where SNRR is the realized signal-to-noise ratio after pulse compression and averaging, also in decibels. The raw SNR can be improved using short-term signal averaging. Averaging $N$ measurements gives realized signal-to-noise ratio of $\mathrm{SNRR}=\mathrm{SNR}+10 \log _{10} N$. For a desired $\varepsilon$ of $0.1 \mathrm{~dB}$ and a reasonable estimated SNR of $26 \mathrm{~dB}$, for example, close to 40 measurements would be needed to achieve the required SNRR of $42 \mathrm{~dB}$.

In the inverse sense of the problem, the required $\varepsilon$ for an effective measurement of $p \mathrm{H}$ from $D_{A}$ can be computed as a function of frequency and range. Expression (13) can be used to obtain SNRR, and SNR and SNRR can be used to obtain $N$. A starting point is the derivative of Eq. (10) given earlier. From the $200-300 \mathrm{~Hz} D_{A}$ curve in Fig. 5 for $L=600 \mathrm{~km}$ and $p \mathrm{H}=7.8$, mean $D_{A}$ is about $1.7 \mathrm{~dB}$ and $\Delta D_{A}$ $=\Delta p \mathrm{H} D_{A} / 0.4343$ is equal to $0.20 \mathrm{~dB}$ for $\Delta p \mathrm{H}=0.05$. To measure this, assume that signal level at each frequency must be determined to an accuracy of $\Delta D_{A} / 4$ (i.e., $\varepsilon$ is required to be less than $0.05 \mathrm{~dB}$ in each band for center frequency 250 $\mathrm{Hz}, L=600 \mathrm{~km}$ ). (These requirements are rough, and can be improved during design of an actual system using statistical methods. They serve the purpose here, yielding approximate measurement requirements versus frequency and range. Absolute numbers of required measurements may vary slightly.) Because $D_{A}$ is a function of range and frequency, the required $\varepsilon(f, L) / \varepsilon(0.25,600)=D A(f, L) / D A(0.25,600)$. Figure $7(\mathrm{c})$ contours $\operatorname{SNRR}(f, L)$ computed directly from these $\varepsilon(f, L)$ using Eq. (13) and the scaling requirements obtained from data of Fig. 5.

Figure 7(d) shows the required number of samples $N(f, L)$ needed for a successful detection of delta- $p \mathrm{H}$ of 0.05 , computed from SNRR (Fig. 7(c)) and SNR (Fig. 7(a)). Two formulas for this are

$$
\begin{aligned}
& \sqrt{N}=\left(\sqrt{2} \times 10^{-\mathrm{SNR} / 20}\right) /\left(10^{\varepsilon / 20}-1\right), \\
& N=10^{\text {(SNRR-SNR }) / 10} .
\end{aligned}
$$

There is a "trough" in the plot showing a low $N$ requirement extending from $[400 \mathrm{~Hz}, 200 \mathrm{~km}]$ to $[250 \mathrm{~Hz}, 800 \mathrm{~km}$ ] (lower center to upper left). The measurement is possible at any of the ranges and frequencies covered in the figure, but the fewest samples are needed at the shortest ranges where SNR is high. If chemical measurement issues demand that longer ranges be used, frequencies of $250-400 \mathrm{~Hz}$ would be best, as indicated by the trough.

To summarize this section, for the inverse problem $(p H$ determination), the balance between the undesirable decrease in SNR with frequency and range, and the desirable $D A$ improvement with increasing range and frequency has been quantified. This is independent of the desirability to propagate long distances to achieve good spatial averaging. 


\section{INTERNAL-WAVE EFFECTS}

Next, frequency-dependent fluctuations of the arrivals that are caused by internal waves are considered. The differencing would reject fluctuations common across the measurement band, but that is a best-case scenario. The worst case is treated here. Many of the fluctuation effects caused by internal waves are somewhat coherent across frequency, but they are treated here as independent at the two frequencies. For fluctuations with normal statistics, the meansquared error of the mean estimate is equal to the variance divided by the number of samples, MSME $=\mu_{2} / N$. This allows fluctuation parameter statistics to be converted to estimates of the quality of sampled means. The presence of internal-wave induced intensity fluctuations is commonly known, and numerous theoretical and computational studies exist. However, the intensity fluctuation variance at $500 \mathrm{~km}$ range is poorly delineated in the literature. This is because difficulties such as inadequate SNR have left comparisons of data with scattering theory unable to fully test the theories.

It is known that an impulsive sound wavefront in the temperate ocean sound channel deforms into an accordion shape (in space at fixed time, and in time at a fixed distance from the source) (Munk et al., 1995). Each leg of the accordion corresponds to a single member of the multi-path arrival structure and to a specific number of upper turning points of the looping sound rays plus an initial direction index (two choices, up or down). Each arrival at a point receiver corresponds to sound that is concentrated along eigenrays connecting source to receiver. The intensity and phase fluctuations of each arrival can be estimated using integrals of ocean properties such as sound-speed anomaly along each ray (Flatté et al., 1979; Flatté and Rovner, 2000). Many of the effects causing intensity (I) fluctuation, such as fluctuating focus effects along the rays due to internal waves, are weakly dependent on frequency. Thus, $D_{A}$ would be only weakly sensitive to them. In the ideal case of infinite SNR and frequency independence of internal-wave effects, a borate absorption measurement could be made with a single measurement of intensity difference at two frequencies, taking only an instant.

However, intensity fluctuations exist which vary across a frequency band on an instantaneous basis. Experiment and computer simulations have shown that internal waves can cause each of the arrivals to be composed of many micromulti-path arrivals (Colosi et al., 1994; Simmen et al., 1997). Interference between these arrivals can cause intensity fluctuations that are stronger functions of frequency than the intensity fluctuations of intact wavefronts because interference is a function of phase, and phase in each band of broadband sound propagated over a fixed path is a function of frequency. The result is that intensity can fluctuate rapidly and independently at two measurement frequencies. Thus, a scintillation in the intensity difference over the two frequencies (which is related to our desired parameter $D_{A}$ via $D_{A}$ $\left.=10 \log _{10}\left(I_{2} / I_{1}\right)\right)$ will result.

Jin and Worcester (1989), using a spectral formalism, computed that about 100 intensity-difference measurements would be needed to suitably average the intensity fluctua- tions in order to achieve a measurement error of $0.3 \mathrm{~dB}$ for $550 \mathrm{~Hz}$, range $750 \mathrm{~km}$ in the North Pacific. The expression is $\varepsilon=10 \log _{10}\left(1+((M-1) / 2 N M)^{1 / 2}\right)$, where $M$ is the number of interfering micro-rays and $N$ is the number of averaged receptions. More averaging would be required to achieve 0.1 dB (see Sec. V C).

There are a few published numbers for the total intensity fluctuation. The scintillation index is defined to be the intensity variance divided by the square of the mean intensity, $S I=\mu_{2} / \mu_{1}^{2}$. This has been observed to be between 0.2 and 2.0 for various resolved early arrivals in a $1000 \mathrm{~km}$ experiment, with an average value of 0.9 (Duda and Bowlin, 1994). These numbers include macro effects of internal waves that are weak functions of frequency, and thus overestimate the fluctuations that should remain after frequency differencing. Values far above 1.0 correspond to strong interference of a few signals, those at 1.0 correspond to saturation (interference of many arrivals), and values less than 0.3 correspond to weak scattering (Born or Rytov) (Flatté et al., 1979). Note that intensity statistics are not normal, but can be approximated as such for the smaller observed values of SI. This is not a severe problem because this is a study of confidence intervals and error bars, and the improvements gained via averaging will be realized nonetheless, although improvement is more difficult to quantify than in the case of normally distributed fluctuations.

It is convenient to obtain a unified expression for the number of measurements needed to obtain the required precision in the presence of the two noise processes: internalwave induced intensity fluctuation and additive Gaussian noise. Define signal waveform as $S(t)=S_{0}(t)+S_{I W}(t)+n(t)$, where $S_{0}$ is mean signal, $S_{I W}$ is additive zero-mean internalwave fluctuation, and $n$ is noise. Using methods in Jin and Worcester (1989) and expressions from Sec. V, one can write for the typical measurement error at each frequency

$$
\varepsilon=20 \log _{10}\left(1+\frac{\left\langle\left|S_{I W}\right|^{2}\right\rangle^{1 / 2}}{\left\langle\left|S_{0}\right|^{2}\right\rangle^{1 / 2}}+\sqrt{2} \times 10^{-(\mathrm{SNR}+10 \log N) / 20}\right) .
$$

The triangle inequality has been used, so this is an upper bound on the deviation between the mean of measured energy $S^{2}$ and the desired energy $S_{0}^{2} \quad$ [i.e., on $\left.10 \log _{10}\left(\left\langle S^{2}\right\rangle /\left\langle S_{0}^{2}\right\rangle\right)\right]$. Now assume that the second term in brackets, the fluctuation amplitude contribution, is reduced with averaging. With normal statistics, the second term becomes $S A / \sqrt{N}$. Our newly defined scintillation amplitude statistic $S A=\left\langle\left|S_{I W}^{2}\right|\right\rangle^{1 / 2} /\left\langle\left|S^{2}\right|\right\rangle^{1 / 2}$ is (oddly) similar in shape to $S I$ for many reasonable pdf's of $S_{I W}$, but there is no simple analytic relationship. For fluctuations with a normal distribution, $S A / S I$ exceeds 1 for low SI but tends to have a value near 0.62 for $S I$ greater than 0.7. SA is about $S I / 2$ for exponentially distributed populations with $S I$ near 1.4. Rearrangement of Eq. (15) yields

$$
\sqrt{N}=\left(S A+\sqrt{2} \times 10^{-\mathrm{SNR} / 20}\right) /\left(10^{\varepsilon / 20}-1\right) .
$$

In the limit of $S A$ going to zero, this expression properly reverts to Eq. (14a) which applies to the case of steady signal with noise. 


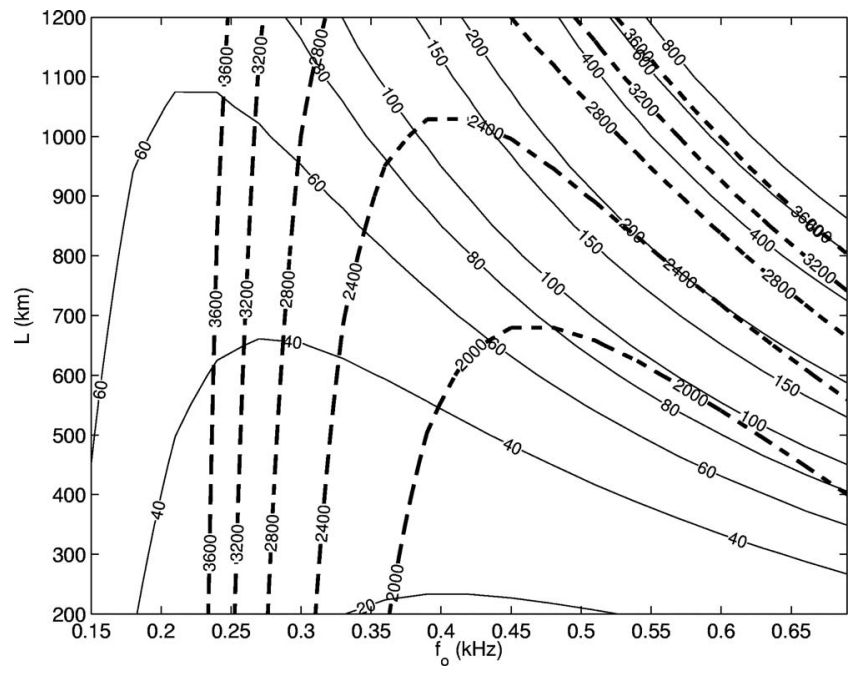

FIG. 8. The required number of samples for $p \mathrm{H}$ measurement with 0.05 precision for the situation of fluctuating signal and noise, and $100 \mathrm{~Hz}$ frequency difference, is contoured over center frequency and range (bold dashed lines). The same quantity for the steady signal and noise situation is also shown (solid lines, repeated from Fig. 5(d)).

The second term of the numerator is typically about 0.11 (22 dB SNR), whereas the scintillation term [for individual wavefront (ray) arrivals] is likely to always exceed this. This means that the signal fluctuations will compromise the measurement ability much more than noise. This agrees with the comparison of the two effects made by Jin and Worcester (1989). The most stringent scenario that they calculate is that 225 measurements would be needed to average over signal fluctuations to reduce error to $0.2 \mathrm{~dB}$. Figure 8 contours the number of samples needed for realistic values of $S A$ and shows that many more than their estimates may be needed. Modeling $S A$ to be linear with range, starting at $S A=0$ at zero range and growing to $S A=1.0$ at $1000 \mathrm{~km}$ range, the plot shows contours of $N$ needed to reduce $\varepsilon$ to the value needed to effectively sample the expected $D A$ to a $p \mathrm{H}$ precision of 0.05 (analogous to procedures of the prior section). These are calculated from Eq. (16). Some of the $N$ contours for the noise-only result of Fig. 7(d) are replotted. The two sets of contours have different shapes, and the fluctuating signal result shows that far more measurements must be averaged than in the noise-only case. There is an order of magnitude difference at range of $800 \mathrm{~km}$ with $600 \mathrm{~Hz}$. For the case that includes fluctuations, there is a "trough" of greatest effectiveness (minimum number of points) passing through $400 \mathrm{~Hz}, 1000 \mathrm{~km}$. If $S A$ is reduced by $50 \%$ (i.e., weaker internal-wave scattering is assumed), the $N$ values fall to about one-third of the values indicated in the contour plot over the area of the trough, which retains its shape. Figure 9 shows results computed in a similar fashion to those of Fig. 8 except a band separation of $200 \mathrm{~Hz}$ is used. The time required to obtain the required order-500 samples can be estimated. For a fluctuation coherence time of $5 \mathrm{~min}$, the time would be about 2 days. As with the $100 \mathrm{~Hz}$ separation situation shown in Fig. 8, reducing SA by $50 \%$ decreases $N$ by about $70 \%$ with little change in the shape of the contours.

There is a possibility that internal-wave induced amplitude fluctuations of distinct arrivals are correlated over fre-

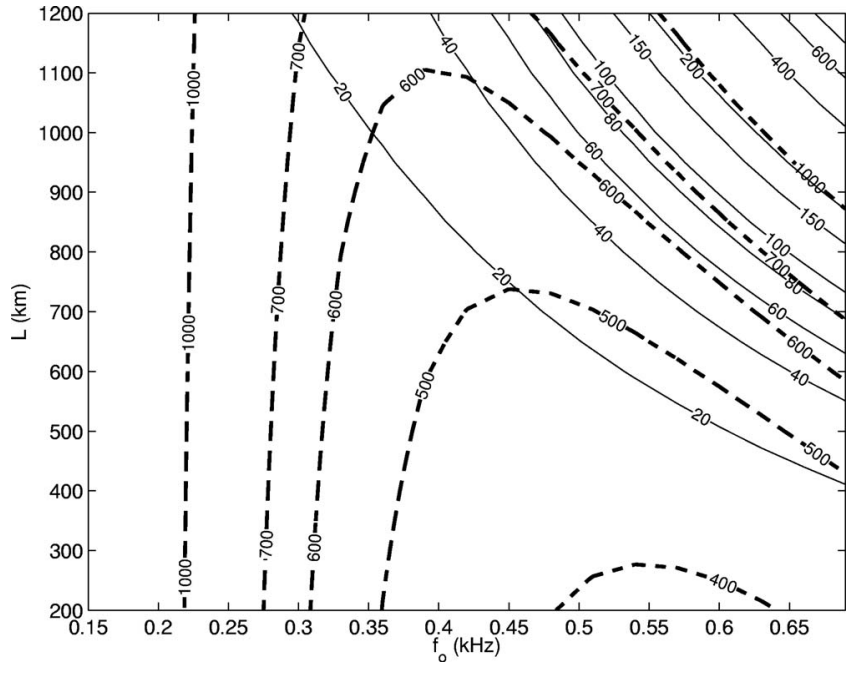

FIG. 9. The number of samples needed to measure $p \mathrm{H}$ to an accuracy of 0.05 units using a $200 \mathrm{~Hz}$ bandwidth measurement is contoured. Dashed contours are for the case that includes signal fluctuations and noise, and the solid contours are for the case of noise only.

quency. In this case it would be advantageous to difference the peak amplitudes of the pulses before averaging (predifferencing). Noise would remain, but fluctuation effects might be reduced or eliminated. An expression for difference $\varepsilon$ can be developed. With noise treated as independent over frequency, the expression will look like Eq. (15) except a difference amplitude statistic can be substituted for $S A$. Because the differencing is done first, the tolerance for $\varepsilon$ for a given delta- $p \mathrm{H}$ is eased with respect to that used earlier by a factor of 2 (i.e., the value is $0.12 \mathrm{~dB}$ for delta- $p \mathrm{H}$ of 0.05 at $600 \mathrm{~km}, 250 \mathrm{~Hz}$ ). The amplitude statistic [second term in Eq. (15)] can take the form $A=\left\langle\left|S_{I W 1}\right|-\left|S_{I W 2}\right|\right\rangle /\left\langle\left|S_{01}\right|-\left|S_{02}\right|\right\rangle$, where subscripts indicate frequency band one or two. Averaging then yields $S A \sqrt{2 / N}$ for the case of uncorrelated fluctuations. This method produces curves similar to those of Fig. 8, but approximately double the numbers of samples indicated in that figure are needed. Thus, adding uncorrelated fluctuations before the averaging process increases their detrimental effect. However, if internal-wave fluctuations are correlated in the two bands, then very improved performance would result. For perfect correlation of internal-wave fluctuations across the band, the noise-only results would hold for the pre-difference technique.

\section{SUMMARY}

The technique of using differential acoustic attenuation measurements at frequencies below $600 \mathrm{~Hz}$ to determine the precise nature of absorption effects (direct measurement) and by extension average $p \mathrm{H}$ (inverse measurement) has been examined. The basic processes are reviewed and the equations are derived. The capabilities of many frequency difference pairs and various acoustic path lengths at providing attenuation measurements of the required accuracy in a noisy environment are examined.

Differential attenuation (energy difference between two frequency bands) might be measurable with equipment of existing types, either using a broad sweep system (Morozov 
and Webb, 2003) or two systems, each with bandwidth of 50 $\mathrm{Hz}$ or more, operating in bands separated by 100 or $200 \mathrm{~Hz}$ (or more). Using established formulas, estimates are made of the required measurement accuracy, in decibels, to make meaningful $p \mathrm{H}$ measurement (inverse problem) for various propagation distances, various center frequencies, and twofrequency band separations. The results are in the form of contours of the quantity of independent samples required to be averaged together to measure effects consistent with $p \mathrm{H}$ fluctuations of 0.05 .

Two scenarios are examined: steady signal (idealized) and fluctuating signal (more realistic). Separated multi-path signal peaks used to estimate energy received after propagating distances of a few hundred kilometers are not steady, but fluctuate, largely due to internal-wave effects. The fluctuations of energy in the field have not been well characterized in the literature. These fluctuations are of order 1 in comparison with the mean energy, so in the high SNR case they dominate the error of the measurement. Conservative estimates of the number of samples required are made assuming the fluctuations are independent in the two frequency bands whose energy is differenced to obtain the measurement. The values are a few thousand for $100 \mathrm{~Hz}$ separation, and order 500 for $200 \mathrm{~Hz}$ separation.

The results reflect a competition between two effects. Attenuation difference grows with range and has a maximum at about $600 \mathrm{~Hz}$. Signal-to-noise ratio shrinks with range and frequency. The result is that paths of $600-1000 \mathrm{~km}$ and frequencies of $375-450 \mathrm{~Hz}$ may be most practical for largescale sensing. These frequencies are below the frequency of maximum sensitivity $(600 \mathrm{~Hz})$, and their effectiveness stems from having better SNR than $600 \mathrm{~Hz}$. Using three rather than two frequencies would provide a slightly better measure of the attenuation curve than would a simple two-frequency difference by adding information.

To confront small open questions regarding the chemistry (Mellen et al., 1983; Zeebe et al., 2001) and the dependencies in Eq. (4), differential-attenuation measurements of absorption can be combined with ship surveys to verify absorption behavior. Regarding the inverse problem $(p \mathrm{H}$ measurement), the method would provide a spatially-averaged measure that is less precise than individual in situ $p \mathrm{H}$ measurements. It would be most useful in two applications. One is in regions of high spatial variability, where unrealistically large numbers of $p \mathrm{H}$ measurement profiles would be required to obtain a good mean estimate. The other application is a time series measurement of $p \mathrm{H}$ variability. This may be most cost-effective in remote regions where a time series involving in situ samples would be difficult. A few hundred transmissions through independent internal-wave fields (a source of noise in the attenuation measurement), possible in a period of a few days, would provide a mean $p \mathrm{H}$ value. Repeat transmission cycles provide the time series. Time series could also be provided by moored autonomous $p \mathrm{H}$ sensors, but fixed-site point sensors may be at risk of not representing large-scale mean $p \mathrm{H}$ conditions very well because of local bias effects. Clearly there are advantages and disadvantages of each measurement technology. Perhaps the strongest potential for the acoustic measure is in remote polar areas, where undersaturation of calcium carbonate minerals may occur first, with strong biological consequences.

\section{ACKNOWLEDGMENTS}

Funding was provided by Woods Hole Oceanographic Institution. Discussions with Bruce Howe and Peter Worcester were helpful. Following suggestions made by the editor and Peter Brewer has significantly improved the manuscript.

Brekhovskikh, L., and Lysanov, Yu. (1982). Fundamentals of Ocean Acoustics (Springer-Verlag, Berlin).

Brewer, P. (1997). "Ocean chemistry of the fossil fuel $\mathrm{CO}_{2}$ signal: The haline signal of "Business as Usual"," Geophys. Res. Lett. 24, 1367-1369. Brewer, P. G., Glover, D. M., Goyet, C., and Shafer, D. K. (1995). "The $p H$ of the North Atlantic Ocean: Improvements to the global model for sound absorption," J. Geophys. Res. 100, 8761-8776.

Caldeira, K., and Wickett, M. E. (2005). "Ocean model predictions of chemistry changes from carbon dioxide emissions to the atmosphere and ocean," J. Geophys. Res. 110, C09S04.

CDIAC (2009a). Carbon Dioxide Information Analysis Center, Environmental Sciences Division, Oak Ridge National Laboratory, U.S. Department of Energy, http://cdiac.ornl.gov/oceans/pacific.html (Last viewed January, 2009).

CDIAC (2009b). Carbon Dioxide Information Analysis Center, Environmental Sciences Division, Oak Ridge National Laboratory, U.S. Department of Energy, http://cdiac.ornl.gov/oceans/glodap/Glopatlas.htm (Last viewed January, 2009).

Colosi, J. A., Flatté, S. M., and Bracher, C. (1994). "Internal-wave effects on 1000-km ocean acoustic pulse propagation: Simulation and comparison to experiment," J. Acoust. Soc. Am. 96, 452-468.

Doney, S. C., Lima, I., Feely, R. A., Glover, D. M., Lindsay, K., Mahowald, N., Moore, J. K., and Wanninkhof, R. (2009b). "Mechanisms governing interannual variability in upper-ocean inorganic carbon system and air-sea $\mathrm{CO}_{2}$ fluxes: Physical climate and atmospheric dust," Deep-Sea Res., Part II (to be published).

Doney, S. C., Tilbrook, B., Roy, S., Metzl, N., Le Quéré, C., Hood, M., Feely, R. A., and Bakker, D. (2009a). "Surface ocean $\mathrm{CO}_{2}$ variability and vulnerability," Deep-Sea Res., Part II (to be published).

Duda, T. F., and Bowlin, J. B. (1994). "Ray-acoustic caustic formation and timing effects from ocean sound-speed relative curvature," J. Acoust. Soc. Am. 96, 1033-1046.

Duda, T. F., Flatté, S. M., Colosi, J. A., Cornuelle, B. D., Hildebrand, J. A., Hodgkiss, W. S., Jr., Worcester, P. F., Howe, B. M., Mercer, J. A., and Spindel, R. C. (1992). "Measured wave-front fluctuations in 1000-km pulse propagation in the Pacific Ocean," J. Acoust. Soc. Am. 92, 939-955. Erbe, C., and Farmer, D. M. (2000). "A software model to estimate zones of impact on marine mammals around anthropogenic noise," J. Acoust. Soc. Am. 108, 1327-1331.

Feely, R. A., Sabine, C. L., Hernandez-Ayon, J. M., Ianson, D., and Hales, B. (2008). "Evidence for upwelling of corrosive "acidified" water onto the continental shelf," Science 320, 1490-1492.

Feely, R. A., Sabine, C. L., Lee, K., Berelson, W., Kleypas, J., Fabry, V. J., and Millero, F. J. (2004). "Impact of anthropogenic $\mathrm{CO}_{2}$ on the $\mathrm{CaCO}_{3}$ system in the oceans," Nature (London) 305, 362-366.

Fisher, F. H., and Simmons, V. P. (1977). "Sound absorption in sea water," J. Acoust. Soc. Am. 62, 558-564.

Flatté, S. M., Dashen, R., Munk, W. H., Watson, K. M., and Zachariasen, F. (1979). Sound Transmission Through a Fluctuating Ocean (Cambridge University Press, London).

Flatté, S. M., and Rovner, G. (2000). "Calculations of internal-waveinduced fluctuations in ocean-acoustic propagation," J. Acoust. Soc. Am. 108, 526-534.

Hester, K. C., Peltzer, E. T., Kirkwood, W. J., and Brewer, P. G. (2008). "Unanticipated consequences of ocean acidification: A noisier ocean at lower $p \mathrm{H}$," Geophys. Res. Lett. 35, L19601.

Jin, G., and Worcester, P. F. (1989). "The feasibility of measuring ocean $p \mathrm{H}$ by long-range acoustics," J. Geophys. Res. 94, 4749-4756.

Liebermann, L. (1949). "Sound propagation in chemically active media," Phys. Rev. 76, 1520-1524.

Mellen, R. H., and Browning, D. G. (1977). "Variability of low-frequency sound absorption in the ocean: $p \mathrm{H}$ dependence," J. Acoust. Soc. Am. 61, 704-706. 
Mellen, R. H., Browning, D. G., and Simmons, V. P. (1981). "Investigation of chemical sound absorption in sea water: Part III," J. Acoust. Soc. Am. 70, 143-148.

Mellen, R. H., Browning, D. G., and Simmons, V. P. (1983). "Investigation of chemical sound absorption in sea water: Part IV," J. Acoust. Soc. Am. 74, 987-993.

Mellen, R. H., Simmons, V. P., and Browning, D. G. (1980). "Lowfrequency sound absorption in sea water: A borate-complex relaxation," J. Acoust. Soc. Am. 67, 341-342.

Morozov, A. K., and Webb, D. C. (2003). "A sound projector for acoustic tomography and global ocean monitoring," IEEE J. Oceanic Eng. 28, 174 185.

Munk, W., Worcester, P., and Wunsch, C. (1995). Ocean Acoustic Tomography (Cambridge University Press, New York).

Orr, J. C., Fabry, V. J., Aumont, O., Bopp, L., Doney, S. C., Feely, R. A., Gnanadesikan, A., Gruber, N., Ishida, A., Joos, F., Key, R. M., Lindsay, K., Maier-Reimer, E., Matear, R., Monfray, P., Mouchet, A., Najjar, R. G., Plattner, G.-K., Rodgers, K. B., Sabine, C. L., Sarmiento, J. L., Schlitzer, R., Slater, R. D., Totterdell, I. J., Weirig, M.-F., Yamanaka, Y., and Yool, A. (2005). "Anthopogenic ocean acidification over the twenty-first century and its impact on calcifying organisms," Nature (London) 437, 681-686.
Simmen, J., Flatté, S. M., and Wang, G.-Y. (1997). "Wavefront folding, chaos, and diffraction for sound propagation through ocean internal waves," J. Acoust. Soc. Am. 102, 239-255.

Takahashi, T., Sutherland, S. C., Feely, R. A., and Wanninkhof, R. (2006). "Decadal change of the surface water $p \mathrm{CO}_{2}$ in the North Pacific: A synthesis of 35 years of observations," J. Geophys. Res. 111, C07S05.

Ternon, J. F., Oudot, C., Gourlaouen, V., and Diverres, D. (2001). "The determination of $p \mathrm{H}_{T}$ in the equatorial Atlantic Ocean and its role in the sound absorption modeling in seawater," J. Mar. Syst. 30, 67-87.

University of Hawaii (2009). School of Ocean an Earth Science and Technology, Hawaii Ocean Timeseries, http://hahana.soest.hawaii.edu/hot (Last viewed January, 2009).

Wootton, J. T., Pfister, C. A., and Forester, J. D. (2008). "Dynamic patterns and ecological impacts of declining ocean $\mathrm{pH}$ in a high-resolution multiyear dataset," Proc. Natl. Acad. Sci. U.S.A. 105, 18848-18853.

Yeager, E., Fisher, F. H., Miceli, J., and Bressel, R. (1973). "Origin of the low-frequency sound absorption in sea water," J. Acoust. Soc. Am. 53, 1705-1707.

Zeebe, R. E., Sanyal, A., Ortiz, J. D., and Wolf-Gladrow, D. A. (2001). “A theoretical study of the boric acid-borate equilibrium in seawater," Mar. Chem. 73, 113-124. 